PAPER

\section{Faraday waves in Bose-Einstein condensates with engineering three-body interactions}

To cite this article: F Kh Abdullaev et al 2016 J. Phys. B: At. Mol. Opt. Phys. 49025302

View the article online for updates and enhancements.
Related content

- Geometric resonances in Bose--Einstein condensates with two- and three-body interactions

Hamid Al-Jibbouri, Ivana Vidanovi, Antun Balaž et al.

- A variational approach to the modulationa instability of a Bose--Einstein condensate Etienne Wamba, Alidou Mohamadou and Timoléon C Kofané

Miscibility in coupled dipolar and nondipolar Bose-Einstein condensates

Ramavarmaraja Kishor Kumar, Paulsamy Muruganandam, Lauro Tomio et al.

\section{Recent citations}

- Lattice and quintic nonlinearity induced
$\frac{\text { stripe phase in Bose-Einstein condensate }}{\text { under non-inertial and inertial motion }}$
Priyam Das
- Faraday Waves in Cold-Atom Systems
with Two- and Three-Body Interactions
Lauro Tomio et al

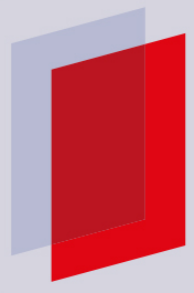

\section{IOP ebooks}

Bringing you innovative digital publishing with leading voices to create your essential collection of books in STEM research. Start exploring the collection - download the first chapter of every title for free. 


\title{
Faraday waves in Bose-Einstein condensates with engineering three-body interactions
}

\author{
F Kh Abdullaev ${ }^{1,2}$, A Gammal ${ }^{3}$ and Lauro Tomio G,4 $^{2,4}$ \\ ${ }^{1}$ Department of Physics, Faculty of Sciences, International Islamic University Malaysia, Bandar Indera \\ Mahkota, Jln. Sultan Ahmad Shah, 25200, Kuantan, Malaysia \\ ${ }^{2} \mathrm{CCNH}$, Universidade Federal do ABC, 09210-170, Santo André, Brazil \\ ${ }^{3}$ Instituto de Física, Universidade de São Paulo, 05508-090, São Paulo, Brazil \\ ${ }^{4}$ Instituto de Física Teórica, UNESP-Universidade Estadual Paulista, 01140-070, São Paulo, Brazil \\ E-mail: tomio@ift.unesp.br
}

Received 11 June 2015, revised 22 September 2015

Accepted for publication 1 October 2015

Published 16 December 2015

\begin{abstract}
We consider Bose-Einstein condensates with two- and three-body interactions periodically varying in time. Two models of time-dependent three-body interactions, with quadratic and quartic dependence on the two-body atomic scattering length $a_{s}$, are studied. It is shown that parametric instabilities in the condensate lead to the generation of Faraday waves (FWs), with wavelengths depending on the background scattering length, as well as on the frequency and amplitude of the modulations of $a_{s}$. From an experimental perspective, this opens a new possibility to tune the period of Faraday patterns by varying not only the frequency of modulations and background scattering length, but also the amplitude of the modulations. The latter effect can be used to estimate the parameters of three-body interactions from the FW experimental results. Theoretical predictions are confirmed by numerical simulations of the corresponding extended Gross-Pitaevskii equation.
\end{abstract}

Keywords: Bose-Einstein condensate, Faraday waves, dynamic properties of condensates, BEC in periodic nonlinear potentials

(Some figures may appear in colour only in the online journal)

\section{Introduction}

The role of three-body interactions in Bose-Einstein condensates (BECs) has attracted a great deal of attention [1-5]. Typically, in dilute systems such as BECs, three-body effects are quite small in comparison with two-body effects. One of the relevant roles that a three-body interaction can play was shown in [6], in the particular case of attractive two-body interactions, where a critical maximum number of atoms exist for stability. The addition of a repulsive three-body potential, even for a very small strength of the three-body interaction, can considerably extend the region of stability. It was also shown in [7] that, if the atom density is considerably high, the three-body interaction can start to play an important role. More recently, a possible interesting scheme for obtaining a condensate with almost pure three-body effects has been suggested in [8]. The idea consists of implementing periodical variations in time of the $s$-wave atomic scattering length $a_{s}$ near zero, such that we have a varying two-body interaction. It can be achieved, for example, by using Feshbach resonance techniques, varying the external magnetic field near the resonance $[9,10]$. Therefore, by considering this procedure, two-body effects can be averaged to zero, enhancing the effective three-body interaction, which is proportional to an even power of the two-body interaction. Note that an analogue of this scheme has been considered before, within an investigation of the role of three-body interactions in arrest collapse in BECs [11]. At the same time, the periodic modulations in time of two- and three-body interactions can lead to parametric instabilities in the ground state, resulting in the generation of Faraday waves (FWs) [12]. The FWs are patterns in BECs which are periodic in space, with the period 
determined by the periodic modulation of trap parameters and strengths of the two- and three-body interactions. The FWs in BECs with two-body interactions have been investigated theoretically in [13], as well as created in cigar-shaped experimental setups [14].

Much attention has been devoted recently to FWs in several investigations. They can be divided into two groups. First, by dealing with the time variation of transverse trap parameters, leading to effective time-dependent nonlinearity in the reduced low-dimensional Gross-Pitaevskii (GP) equation. As examples with oscillating transverse frequency of the trap, we can mention the following recent studies: lowand high-density one-component BECs [13, 15-18]; twocomponent BECs in trap with modulated transverse confinement $[15,19]$; Fermi superfluids in BECs with two- and threebody interactions [20]; and Fermi superfluids at zero temperature [21], where the FWs were considered as a relevant tool to study Bardeen-Cooper-Schrieffer/BEC crossover. A second group of investigations has used the modulation in time of the strength of the interaction. As an example, we have two-component BECs with time-dependent inter- and intra-species interactions, with single and coupled BECs with time-dependent dipolar interactions, where FWs are considered an excellent tool to study nonlocal effects in polar gases [22, 23]. Another example is given in [24], considering studies of superfluid Bose-Fermi mixtures with modulated two-body scattering length for the Bose-Fermion system. Also belonging to this group are studies with an analysis of parametric instabilities in an array of BECs with varying atomic scattering length, based on a discrete nonlinear Schrödinger equation [25]. One should also observe that an analogue of FW patterns can also be found in optical fibre systems [26-28].

By taking into consideration effects due to three-body interactions in BECs, an important point that one should consider is that three-body effects are defined by the value of the two-body interaction (atomic scattering length). Therefore, by varying the scattering length in time, the three-body interaction will also be affected, with the functional form being defined by the corresponding physical model. By taking into account this dependence, one can expect new peculiarities in the FW generation in BECs with two- and three-body interactions. With this motivation, we are concerned in the present paper with an investigation of FW generation in BECs by considering two possible regimes leading to the modulation of the three-body parameter. First, motivated by a model presented in [8], we analyse the case when the strength of the three-body interaction is proportional to the square of the twobody scattering length. In such a model, the corresponding GP type of equation has a term that mimics three-body interactions, which appears in the description of high-density BECs in cigar-type traps $[29,30]$. A second possibility for the modulation of the three-body parameter can arise by considering the case of large two-body scattering lengths near the Efimov regime [31], where the number of three-body states (resonant or bound) increases as the energy of the two-body system goes to zero. In such cases, the strength of the three- body interaction is predicted to be proportional to the fourth power of the atomic scattering length [3, 4].

In both the cases we have analysed, we observe that the FW parameters depend additionally on the amplitude of the time modulations of the atomic scattering length, and not just on the corresponding frequency and background two-body scattering length, such that one can experimentally tune the wavelength of FW. In this way, from the amplitude of the modulations necessary to experimentally obtain the FW patterns one can also estimate the two- and three-body interaction parameter.

\section{Model}

Let us consider a quasi-one-dimensional (1D) Bose-Einstein condensate with atoms of mass $m$, with two- and three-body interactions varying in time. The system is described by a 1D time-dependent Gross-Pitaevskii equation (GPE), with cubic and quintic terms parametrised, respectively, by the functions $\Gamma(t)$ and $G(t)$. By also considering a possible time-independent external interaction $V_{\text {ext }}$, with the wave-function $\psi \equiv \psi$ $(x, t)$ normalized to the number of atoms $N$, the equation is given by

$$
\begin{aligned}
\mathrm{i} \hbar \frac{\partial \psi}{\partial t}= & -\frac{\hbar^{2}}{2 m} \frac{\partial^{2} \psi}{\partial x^{2}} \\
& +V_{\text {ext }}(x) \psi-\Gamma(t)|\psi|^{2} \psi-G(t)|\psi|^{4} \psi,
\end{aligned}
$$

where $\Gamma(t)$ is related linearly with the two-body $s$-wave atomic scattering length $a_{s}(t)$, which can be varied in time by considering Feshbach resonance techniques [9]. The possible ways that the three-body strength $G(t)$ can be varied in time will depend on specific atomic characteristics, which are also related to the type of two-body interaction, as well as induced by some external interactions acting on the condensate.

Several examples can be considered, following equation (1), which can be rewritten with dimensionless quantities [32], by changing the space-time variables such that $t \rightarrow t / \omega_{\perp}$ and $x \rightarrow x 1_{\perp}$, where we have a length scale $l_{\perp}$ and a transverse frequency $\omega_{\perp}$ related by $l_{\perp} \equiv \sqrt{\hbar / 2 m \omega_{\perp}}$. Therefore, in the new dimensionless quantities, with $u \equiv u(x, t)=\sqrt{l_{\perp}} \psi$ and $V_{\text {ext }}=\hbar \omega_{\perp} \mathcal{V}_{\text {ext }}$, we have

$\mathrm{i} \frac{\partial u}{\partial t}+\frac{\partial^{2} u}{\partial x^{2}}-\mathcal{V}_{\mathrm{ext}}(x) u+\gamma(t)|u|^{2} u+g(t)|u|^{4} u=0$,

where the dimensionless time-dependent two- and three-body parameters are, respectively, given by

$$
\gamma(t) \equiv \sqrt{\frac{2 m}{\hbar^{2}}} \frac{\Gamma(t)}{\sqrt{\hbar \omega_{\perp}}} \text { and } g(t) \equiv \frac{2 m}{\hbar^{2}} G(t) .
$$

In the following expressions, we consider that no external potential is applied to the system $\left(\mathcal{V}_{\text {ext }}=0\right)$, such that the natural scale is the $s$-wave two-body scattering length $a_{s}$ at $t=0$, which will define $\omega_{\perp}$ and the corresponding length $l_{\perp}=2 a_{s}$. 
First, in the present work, we consider a non-dissipative system, such that $\gamma(t)$ and $g(t)$ are real. Next, the existence of dissipation due to three-body recombination processes is also studied by changing the definition of $g(t)$ to a more general form, where the dissipation is parameterised by a constant $\kappa_{3}$, such that

$$
g_{c}(t)=g(t)+\mathrm{i} \kappa_{3} .
$$

The different scenarios of the time modulations for the twoand three-body interactions can be exemplified by the following models:

1. Three-body interaction proportional to $\left[a_{s}(t)\right]^{2}$ (quadratic case). This case can occur in a model for a BECs with a 1D non-polynomial GP equation, confined in a cigar-type trap [29]. By a series expansion, valid for small $a_{s}|\psi|^{2}$, an effective quintic parameter can be derived in equation (1), which is given by $G(t) \equiv 2 \hbar \omega_{\perp} a_{s}^{2}(t)$. A similar form of the corresponding equation, for a cigar-type trap, was also derived in [30]. A quadratic dependence of $G(t)$ on $a_{s}(t)$ can also occur in the case when $\Gamma \equiv \Gamma(x, t) \approx \cos (\omega t) \cos (k x)$, corresponding to a time-dependent short-scale nonlinear optical lattice. In this case, averaged over short-scale modulations in space, the dynamics are described by a GP equation with effective time-dependent three-body interactions $[33,34]$. Another model with quadratic dependence on $a_{s}$ was also suggested in [8], considering effective threebody interactions for atoms loaded in a deep optical lattice.

2. Three-body interaction proportional to $\left[a_{s}(t)\right]^{\mathbf{4}}$ (quartic case). By varying $a_{s}(t)$ through the Feshbach resonance techniques, as the absolute value of this twobody observable becomes very large, one approaches the unitary limit $\left(\left|a_{s}\right| \rightarrow \infty\right)$ where many three-body bound states and resonances can be found. This behaviour will induce changes in the corresponding quintic parameter of the GP equation, such that in equation (1) we have $G(t) \sim a_{s}^{4}(t)$ [3].

\section{Modulational instability}

In this section we consider a modulational instability (MI) of the nonlinear plane-wave solution for the equation (2), such that

$$
\begin{aligned}
u_{0} \equiv u(0, t) & =A e^{\mathrm{i} \theta(t)} \\
\text { where } \theta(t) & =A^{2} \int_{0}^{t}\left[\gamma\left(t^{\prime}\right)+A^{2} g\left(t^{\prime}\right)\right] \mathrm{d} t^{\prime}
\end{aligned}
$$

To analyse MI we will look for a solution of the form

$$
u(x, t)=[A+\delta u(x, t)] e^{\mathrm{i} \theta(t)}, \text { with } \delta u \ll A .
$$

By substituting the above expressions in equation (2) and keeping only linear terms $\delta u \equiv \delta u(x, t)$, we have

$$
\mathrm{i} \frac{\partial \delta u}{\partial t}+\frac{\partial^{2} \delta u}{\partial x^{2}}+A^{2}\left[\gamma(t)+2 A^{2} g(t)\right]\left(\delta u+\delta u^{*}\right)=0
$$

Now, by introducing $\delta u=v+\mathrm{i} w$, where $v \equiv v(x, t)$ and $w \equiv$ $w(x, t)$, and going to the corresponding Fourier components, $V$ $\equiv V(k, t)$ and $W \equiv W(k, t)$, according to

$$
(v, w)=\int e^{\mathrm{i} k x}(V, W) \mathrm{d} k,
$$

we obtain the system of equations:

$$
\begin{aligned}
\frac{\mathrm{d} V}{\mathrm{~d} t} & =k^{2} W, \\
\frac{\mathrm{d} W}{\mathrm{~d} t} & =-k^{2} V+2 A^{2}\left[\gamma(t)+2 A^{2} g(t)\right] V .
\end{aligned}
$$

Finally we have

$$
\frac{\mathrm{d}^{2} V}{\mathrm{~d} t^{2}}+k^{2}\left[k^{2}-2 A^{2}\left(\gamma(t)+2 A^{2} g(t)\right)\right] V=0 .
$$

\subsection{Influence of the inelastic three-body collisions}

By taking into account inelastic three-body collisions, defined by a dimensionless parameter $\kappa_{3}$, one should add the term $\mathrm{i} \kappa_{3}|u|^{4} u$ in equation (2). In this case, by replacing $g(t)$ with $g_{c}(t)=g(t)+\mathrm{i} \kappa_{3}$, the equations (5) and (6) have to be replaced by

$$
\begin{gathered}
u(x, t)=[A(t)+\delta u(x, t)] e^{\mathrm{i} \theta(t)}, \\
A(t)=A_{0}\left(1+4 \kappa_{3} A_{0}^{4} t\right)^{-1 / 4}, \\
\theta(t)=\int_{0}^{t} \mathrm{~d} s\left[\gamma(s) A^{2}(s)+g(s) A^{4}(s)\right] .
\end{gathered}
$$

In the above expression for $\theta(t)$, we neglect $\delta u(x, t)$ with the assumption that $A(t) \gg \delta u(x, t)$. Next, by following the procedure of the previous subsection, with $\delta u=v+\mathrm{i} w$, for the Fourier component $V$ we obtain

$$
\begin{gathered}
\frac{\mathrm{d}^{2} V}{\mathrm{~d} t^{2}}+k^{2}\left[k^{2}-2 A(t)^{2}\left(\gamma(t)+2 A(t)^{2} g(t)\right)\right] V \\
=-6 \kappa_{3} A(t)^{4} \frac{\mathrm{d} V}{\mathrm{~d} t}+15\left[\kappa_{3} A(t)^{4}\right]^{2} V .
\end{gathered}
$$

Therefore, due to inelastic three-body collisions, in equation (10) we have the additional dissipative term $6 \kappa_{3}$ $A^{4} \mathrm{~d} V / \mathrm{d} t$, together with a term $\sim \kappa_{3}^{2}$, which can be neglected for small $\kappa_{3}$. This will lead to the appearance of the threshold in the amplitude of modulations of the scattering length for the existence of the parametric resonances.

\subsection{Model of three-body interactions with quadratic dependence on the scattering length}

3.2.1. Modulational instability for periodic variations of the scattering length. Next, in this subsection, we consider the MI for the case of periodic modulations of the scattering length in time, given by $\gamma(t)$, with the three-body interaction 
term, $g(t)$, having a quadratic dependence on $\gamma(t)$ :

$$
\gamma(t)=\gamma_{0}+\gamma_{1} \cos (\omega t), g(t)=c\left[\gamma_{0}+\gamma_{1} \cos (\omega t)\right]^{2},
$$

where $\gamma_{0}$ refers to the natural two-body scattering length, which can be attractive $\left(\gamma_{0}>0\right)$ or repulsive $\left(\gamma_{0}<0\right)$, and $\gamma_{1}$ is the amplitude of the periodic modulation, such that we can take it as a positive quantity. We should note that $[1,6]$ are mainly concerned with three-body repulsion as a way to stabilise a condensate with attractive two-body interaction. However, in the present case, as we are interested in examining the emergence of $\mathrm{FW}$ patterns, we consider interesting conditions where the time-dependent parameter $g$ $(t)$, given by equation (14), is positive $(c>0)$, implying in attractive three-body interaction.

By considering equation (14), without dissipation $\left(\kappa_{3}=0\right)$, from equation (10) we obtain

$$
\frac{\mathrm{d}^{2} V}{\mathrm{~d} t^{2}}+\Omega^{2}\left[1-f_{1} \cos (\omega t)-f_{2} \cos (2 \omega t)\right] V=0,
$$

where

$$
\begin{aligned}
\Omega^{2} & \equiv k^{2} \Delta \equiv k^{2}\left\{k^{2}-2 A^{2}\left[\gamma_{0}+A^{2} c\left(2 \gamma_{0}^{2}+\gamma_{1}^{2}\right)\right]\right\}, \\
f_{1} & \equiv \frac{2 \gamma_{1} A^{2}\left(1+4 c A^{2} \gamma_{0}\right)}{\Delta}, f_{2} \equiv \frac{2 c \gamma_{1}^{2} A^{4}}{\Delta} .
\end{aligned}
$$

We have parametric resonances for two cases, at $\omega=2 \Omega(\eta \equiv$ 1) and $\omega=\Omega(\eta \equiv 2)$, such that the corresponding wavenumber $k_{F}^{\eta}$ is given by

$$
\begin{aligned}
& k_{F}^{(\eta)}= \pm \sqrt{\frac{M_{ \pm}}{2}+\frac{1}{2} \sqrt{M_{ \pm}^{2}+(\eta \omega)^{2}}} \equiv L_{\eta}, \text { with } \\
& M_{ \pm} \equiv 2 A^{2}\left[ \pm\left|\gamma_{0}\right|+A^{2} c\left(2 \gamma_{0}^{2}+\gamma_{1}^{2}\right)\right],
\end{aligned}
$$

where $M_{+}$is for attractive or zero two-body interactions, $\gamma_{0} \geqslant$ 0 , and $M_{-}$is for the repulsive case, $\gamma_{0}<0$. In the present case, as we are analysing the case with $c>0, M_{-}$can be set to zero or negative only for repulsive interactions. In the following, we consider only the relevant positive sign for the resonance wavenumber $k_{F}$.

1. Let us consider more explicitly the first resonance, $\omega=2 \Omega(\eta=1)$. In the attractive or zero two-body interactions, $\gamma_{0} \geqslant 0, M_{+}>0$, the wavenumber $k_{F}^{1}$ (corresponding to a length $L_{1}$ ) of the Faraday pattern, is such that

$$
k_{F}^{(1)} \equiv \frac{2 \pi}{L_{1}}=\sqrt{\frac{M_{+}}{2}\left(\sqrt{1+\frac{\omega^{2}}{M_{+}^{2}}}+1\right)}
$$

where $L_{1}$ gives the period of the generated Faraday pattern in space. For large frequencies of modulations, with $\omega \gg M_{+}$, the period behaves like $L_{1} \sim 1 / \sqrt{\omega}$. In the case of repulsive two-body interactions, $\gamma_{0}<0$, which can be more easily explored in experiments, we have two possibilities, with $M_{-}$positive or negative. When $M_{-}$is positive, we can use the same expression for $k_{F}$ as in equation (18) with $M_{+}$replaced by $M_{-}$.
However, for such repulsive interaction, $M_{-}<0$ can only be satisfied if $\gamma_{1}<1 /\left(2 \sqrt{2} c A^{2}\right)$ and $\left|\gamma_{0}\right|$ is within the interval

$$
\begin{aligned}
& 1+\sqrt{1-2\left(2 c A^{2} \gamma_{1}\right)^{2}}>\left(4 c A^{2}\left|\gamma_{0}\right|\right) \\
& >1-\sqrt{1-2\left(2 c A^{2} \gamma_{1}\right)^{2}} \text {. }
\end{aligned}
$$

If these conditions are satisfied, the Faraday patterns are given by

$$
k_{F_{-}}^{(1)} \equiv \frac{2 \pi}{L_{1}}=\sqrt{\frac{\left|M_{-}\right|}{2}\left(\sqrt{1+\frac{\omega^{2}}{M_{-}^{2}}}-1\right)} .
$$

However, we should note that the above conditions with (19) are too restrictive for experimental observation. Instead, in the repulsive case $\left(\gamma_{0}<0\right)$, one should search FW patterns outside this limit, when equation (18) can be applied. In figure 1, we show the behaviour of the period of the oscillations as a function of the amplitude $\gamma_{1}$, for two cases of repulsive interactions, with fixed $\gamma_{0}=-0.5$ and -1 .

2. For the second parametric resonance, $\omega=\Omega(\eta=2)$, the corresponding pattern is only due to the three-body effects. In this case, by following equation (17), we obtain the same expressions (18) and (20) for $\eta=2$, as in the first case shown above, with replacement of $\omega$ by $2 \omega$. For the period, we have $L_{1}=\sqrt{2} L_{2}$.

From equations (18) and (20), we observe the existence of an additional dependence on the wavenumber of the Faraday pattern from the amplitude of modulations $\gamma_{1}$. This result is new, as far as we know, since in previous investigations [13-15] $k_{F}$ is independent of $\gamma_{1}$. For large $\gamma_{1} \gg 1$ we have estimated that $L_{F} \sim 1 / \gamma_{1}$. Thus, by varying $\gamma_{1}$ and with the knowledge of the effective parameters for the two- and three-body interactions, one can tune the corresponding period of the Faraday pattern.

3.2.2. Modulational instability for fast periodic variations of the scattering length. Let us consider the case of strong fast modulations, when $\omega, \gamma_{1} \gg 1$ and $\gamma_{1} / \omega \sim O(1)$. Following $[11,35]$, it is useful to perform the following change of variables:

$$
\begin{aligned}
u(x, t)= & U(x, t) \exp \left(\mathrm{i} \Gamma_{1}(t)|U(x, t)|^{2}\right. \\
& \left.+\mathrm{i} \Gamma_{2}(t)|U(x, t)|^{4}\right),
\end{aligned}
$$

where $\Gamma_{1}(t)$ and $\Gamma_{2}(t)$ are antiderivatives of $\gamma_{1}(t)$ and $g(t)$, respectively:

$$
\Gamma_{1}(t)=\int_{0}^{t} \mathrm{~d} s \gamma_{1}(s) \text { and } \Gamma_{2}(t)=\int_{0}^{t} \mathrm{~d} s g(s) .
$$

$U \equiv U(x, t)$ is a slowly varying function of $x$ and $t$. To find out the GP equation, averaged over a period of fast oscillations, we first obtain the averaged Hamiltonian. By substituting the equation (21) into the expression for the Hamiltonian and by 

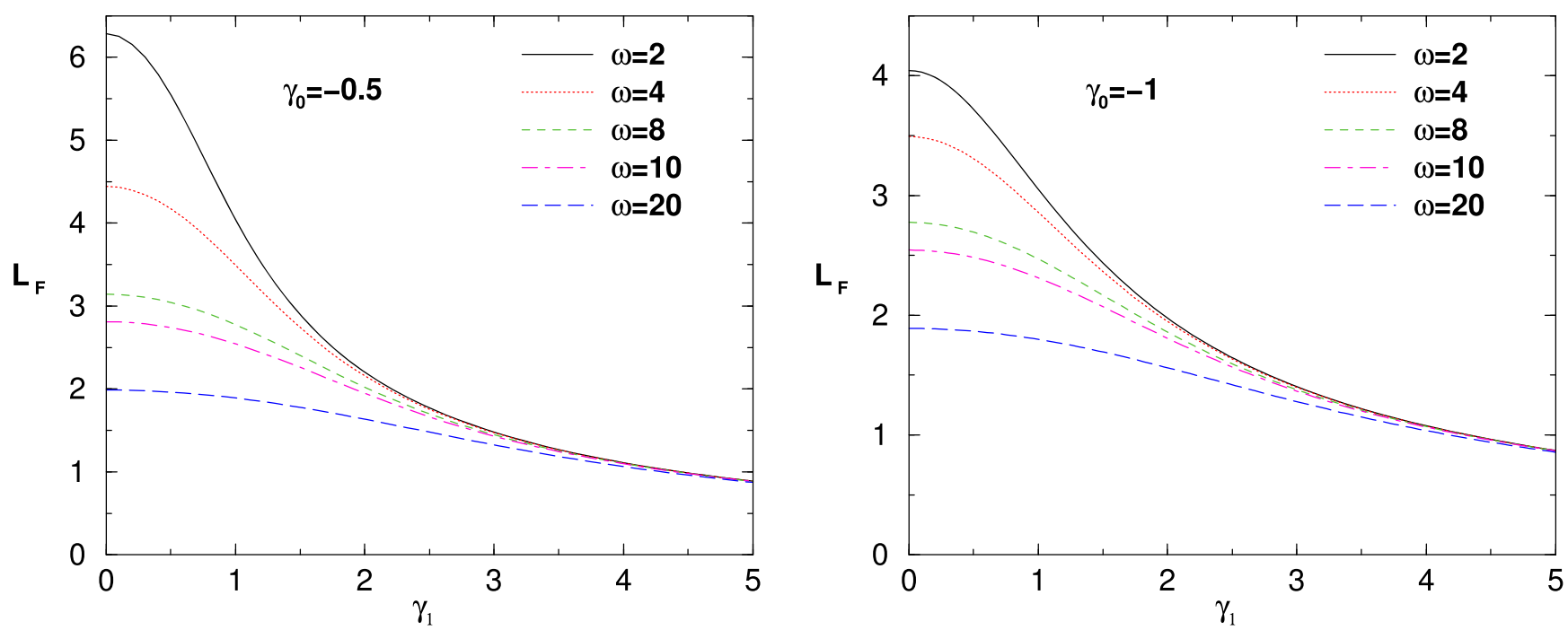

Figure 1. When the three-body interaction is proportional to $\left[a_{s}(t)\right]^{2}$, given by equation (14), we show the behaviour of the period of FW oscillations, $L_{F}=L_{1}$ in case $2 \Omega=\omega$, and $=L_{2}$, when $\Omega=\omega$ ), given as functions of $\gamma_{1}$, for a few set of frequencies $\omega$ and for two cases of two-body repulsive interactions. All quantities are dimensionless and we fix the other parameters such that $A=c=1$.
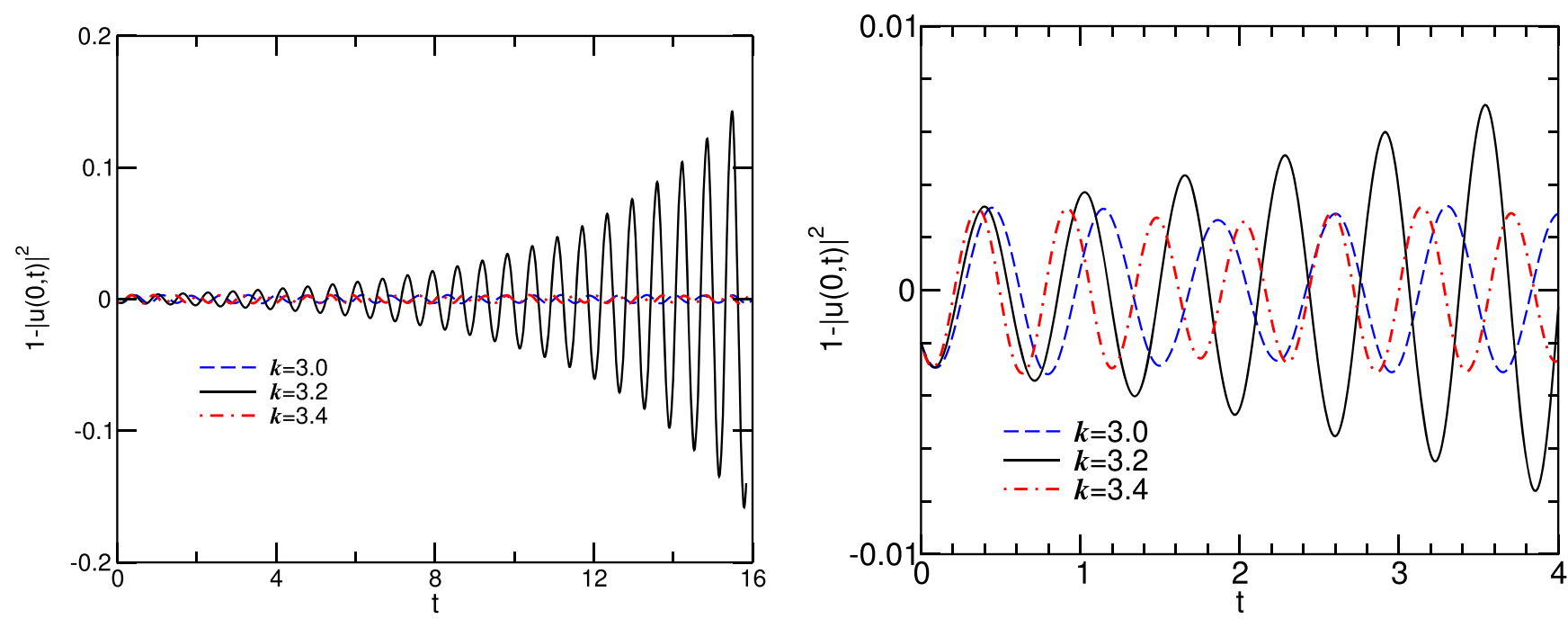

Figure 2. Behaviour of the central density $|u(0, t)|^{2}$, as a function of time, showing the emergence of the first parametric resonance (for $\omega=20$ ), from full numerical calculations. In full agreement with analytical predictions for the values of $k$, the resonance for $k=k_{F}=3.2$ is obtained. The other parameters, in this case, are such that $\gamma_{0}=0, \gamma_{1}=0.5, \epsilon_{0}=0.001, A=1$, and $c=1$, with all quantities in dimensionless units. In the right frame, we show a smaller time interval $(t<4)$ for a clear identification of the plots for the given values of $k$.

averaging over the period of rapid oscillations, we obtain

$$
\begin{aligned}
H_{\mathrm{av}}= & \int_{-\infty}^{\infty} \mathrm{d} x \mathcal{H}_{\mathrm{av}} \\
\mathcal{H}_{\mathrm{av}}= & \left\{\left|\frac{\mathrm{d} U}{\mathrm{~d} x}\right|^{2}+\left[\sigma_{1}^{2}\left(\frac{\mathrm{d}|U|^{2}}{\mathrm{~d} x}\right)^{2}\right.\right. \\
& \left.\left.+\sigma_{2}^{2}\left(\frac{\mathrm{d}|U|^{4}}{\mathrm{~d} x}\right)^{2}\right]|U|^{2}-\frac{g_{\text {eff }}}{3}|U|^{6}\right\},
\end{aligned}
$$

where $\sigma_{1}^{2} \equiv \frac{c \gamma_{1}^{2}}{2 \omega^{2}}$

$$
\sigma_{2}^{2} \equiv \frac{c \gamma_{1}^{4}}{32 \omega^{2}}, g_{\mathrm{eff}} \equiv c \frac{\gamma_{1}^{2}}{2}
$$

The averaged GP equation is given by

$$
\begin{aligned}
\mathrm{i} \frac{\partial U}{\partial t}= & \frac{\delta \mathcal{H}_{\mathrm{av}}}{\delta U^{*}} \\
= & -\frac{\mathrm{d}^{2} U}{\mathrm{~d} x^{2}}-\left(\sigma_{1}^{2}+16 \sigma_{2}^{2}|U|^{4}\right)\left[\left(\frac{\mathrm{d}|U|^{2}}{\mathrm{~d} x}\right)^{2}\right. \\
& \left.+2|U|^{2} \frac{\mathrm{d}^{2}|U|^{2}}{\mathrm{~d} x^{2}}\right] U-g_{\text {eff }}|U|^{4} U .
\end{aligned}
$$

Performing standard MI analysis with the averaged equation (24), i.e. considering the evolution of the perturbed 

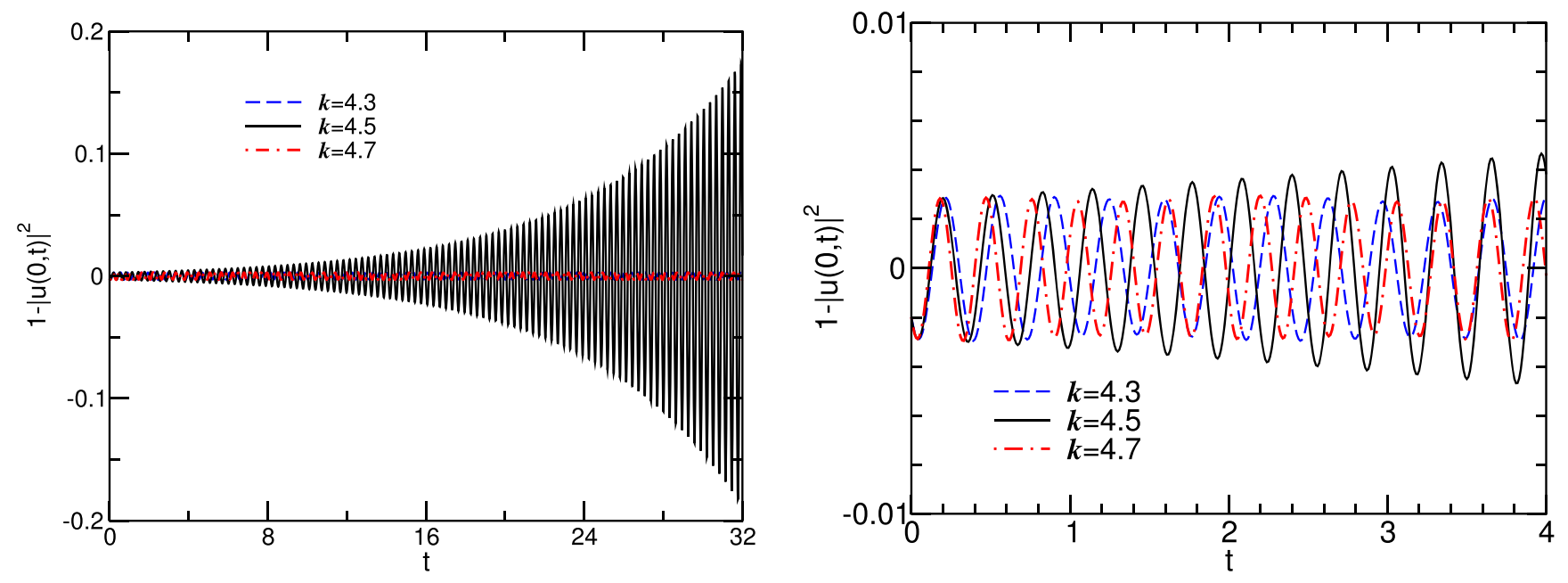

Figure 3. Following figure 2 the behaviour of central density is displayed as a function of time, showing the emergence of the second parametric resonance $(\omega=40)$, from full numerical calculations. In this case, again in agreement with the analytical prediction, the resonance occurs at $k=4.5$. In the right frame, for $t<4$, we also show the plots for the given $k$, in order to appreciate how the resonance starts to appear. The other parameters are the same as in figure 2 .

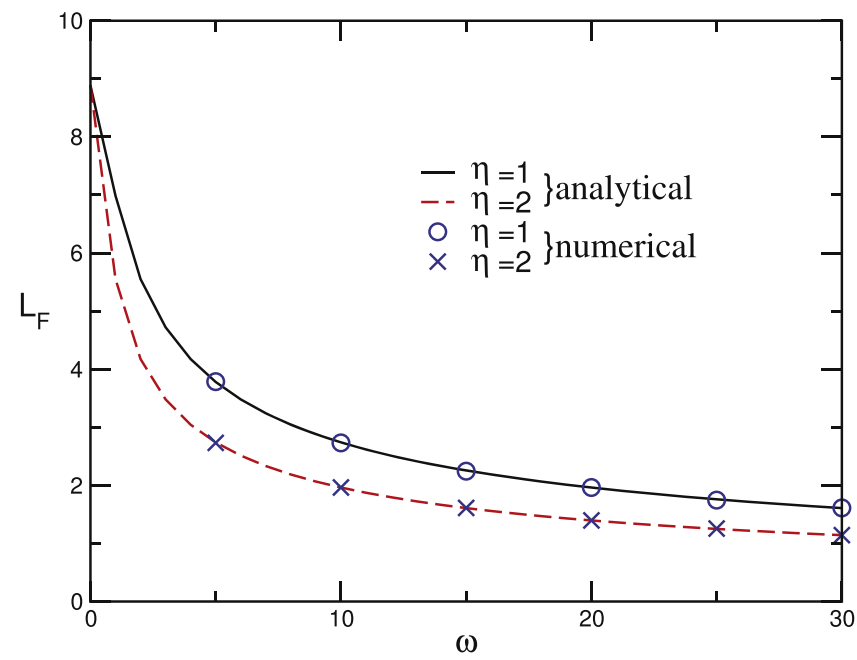

Figure 4. The length of Faraday pattern, $L_{F}$, is presented as a function of the frequency, for the first $(\eta=1)$ and second $(\eta=2)$ resonances, by considering analytical (solid and dashed lines) and numerical results (empty circle and crosses). As shown, we have a perfect agreement between analytical and numerical results. $L_{F}$ and $\omega$ are in dimensionless units.

nonlinear plane-wave solution in the form:

$$
U=(A+\delta U(x, t)) e^{\mathrm{i} g_{\mathrm{eff}} A^{4} t} .
$$

Next, with $\delta U \equiv P+\mathrm{i} Q$, by performing the corresponding Fourier transforms $\quad\left[P=\int \mathrm{d} k p(k) \exp (\mathrm{i} k x) \quad\right.$ and $\left.Q=\int \mathrm{d} k q(k) \exp (\mathrm{i} k x)\right]$, we obtain the dispersion relation

$$
\begin{aligned}
\Omega_{\mathrm{av}}^{2} & =k^{2}\left[(1+r) k^{2}-4 g_{\mathrm{eff}} A^{4}\right], \\
\text { where } r & \equiv 2 A^{4}\left(\sigma_{1}^{2}+4 \sigma_{2}^{2} A^{4}\right) .
\end{aligned}
$$

From this expression, the maximum gain occurs at the wavenumber

$$
k_{c}=\frac{\sqrt{2 g_{\mathrm{eff}}} A^{2}}{\sqrt{1+r}},
$$

with the corresponding gain rate given by

$$
p_{c}=\frac{2 \sqrt{g_{\mathrm{eff}}} A^{4}}{\sqrt{1+r}} .
$$

Note that management of the scattering length can suppress the MI and will correspond to a weakening of the effective three-body interactions. This effect leads to arrest of collapse and makes possible the existence of stable bright matter wave solitons in BECs with effective attractive quintic nonlinearity (see also [36]).

The procedure of averaging out two-body processes, with enhancement of the three-body (attractive) interactions, in the quasi-1D geometry, can lead to the collapse, which will happen when the number of atoms $N$ exceeds a critical value $N_{\mathrm{c}}$. However, for the strong and rapid modulations case, as we verify from the averaged equation (24), a nonlinear dispersion term appears. This term showed that for small widths the effective repulsion can arrest the collapse, such that stable bright solitons can exist for $N>N_{\mathrm{c}}$. This problem requires a separate investigation.

\subsection{Model of three-body interactions with quartic dependence on the scattering length}

Let us now consider the case when the strength of three-body interactions is proportional to the quartic power of the scattering length; i.e., when $g(t) \sim a_{s}^{4}$ [3], such that

$\gamma(t)=\gamma_{0}+\gamma_{1} \cos (\omega t), g(t)=c_{E}\left[\gamma_{0}+\gamma_{1} \cos (\omega t)\right]^{4}$. 

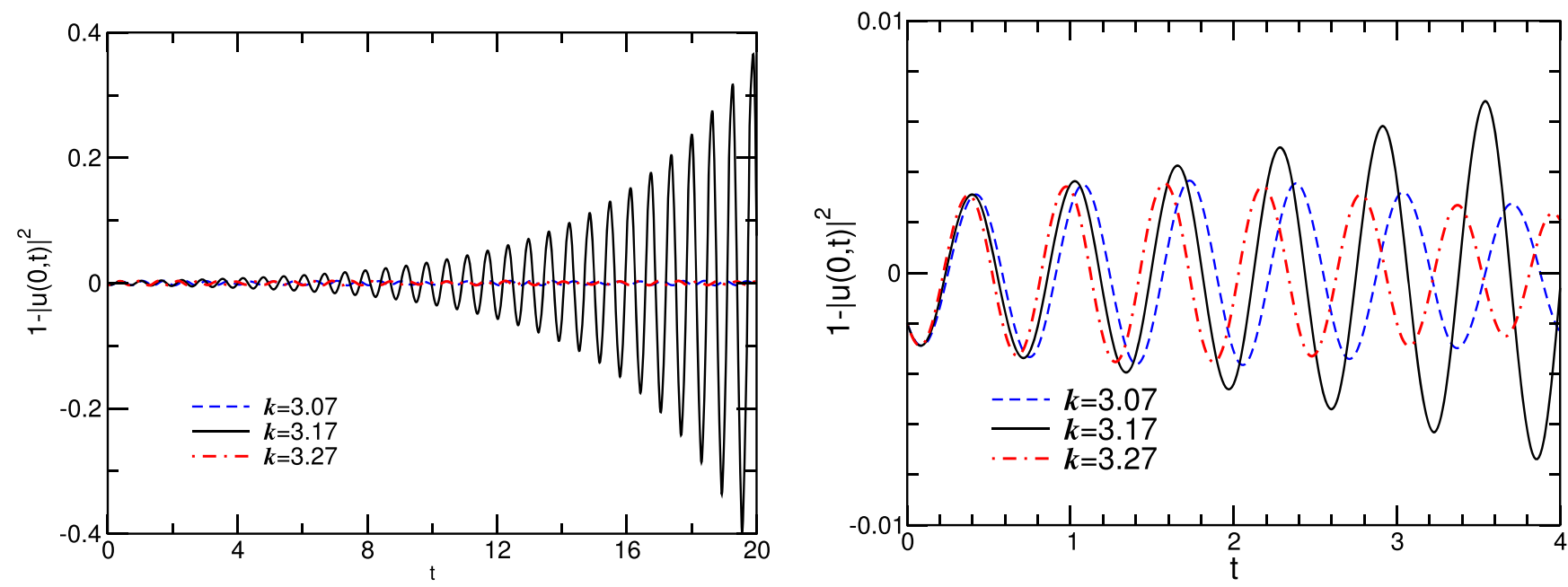

Figure 5. For the case when the behavior of three-body interactions goes with the fourth power of the two-body interactions, in analogy with figures 2 and 3, we present the behaviour of central density, as a function of time, showing the emergence of the first parametric resonance (for $\omega=20$ ), from full numerical calculations. As before, we follow the analytical prediction, obtaining the resonance for $k=3.17$. As in previous figures, the other parameters, in dimensionless units, are such that $\gamma_{0}=0, \gamma_{1}=0.5, \epsilon_{0}=0.001, A=1$, and $c=1$. We also present a plot, in the right frame, considering a smaller interval $t<4$.
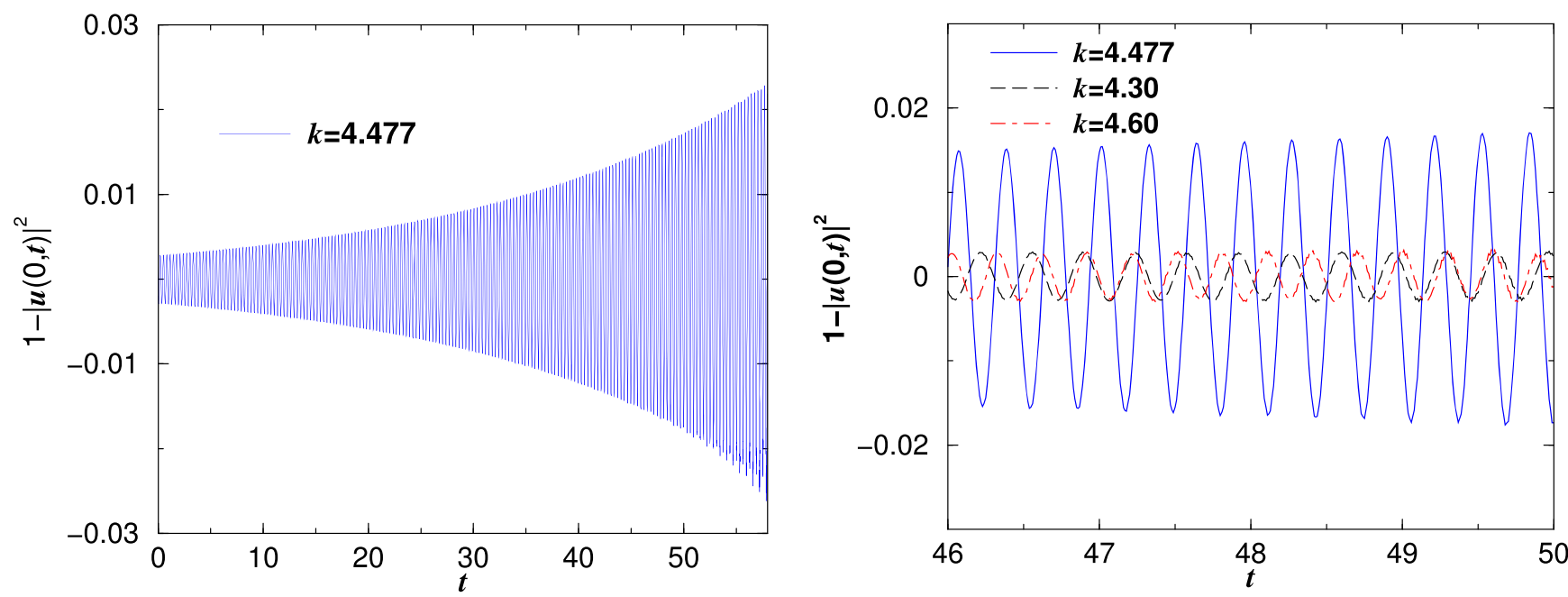

Figure 6. Following the results presented in figure 5, here we consider the second parametric resonance $(\omega=40)$, from full numerical calculations. As before, we follow the analytical prediction for the resonance, which is close to $k=4.477$. We note, in this case, that the rate of increasing of the amplitude at the resonance is not so fast as in the case of the first resonance. In the right panel we present two different small time intervals, where we are comparing the results obtained at the resonance with two other values of $k$. As before, the other parameters are in dimensionless units and such that $\gamma_{0}=0, \gamma_{1}=0.5, \epsilon_{0}=0.001, A=1$, and $c=1$.

The possibility of attractive or repulsive three-body interaction will correspond, respectively, to $c_{E}$ being positive or negative, which can happen for attractive or repulsive twobody interactions. In the next section, in our numerical search for the FW patterns near the Efimov limit, both cases are verified.

From equation (13) without the term $\sim \kappa_{3}^{2}$, the expression for $V \equiv V(k, t)$ is given by

$$
\frac{\mathrm{d}^{2} V}{\mathrm{~d} t^{2}}+\Omega_{1}^{2}\left[1-\sum_{j=1}^{4} h_{j} \cos (j \omega t)\right] V+6 \kappa_{3} A^{4} \frac{\mathrm{d} V}{\mathrm{~d} t}=0
$$

where

$$
\left.\begin{array}{rl}
\Omega_{1}^{2} & \equiv k^{2}\left(k^{2}-2 A^{2} a_{0}\right), \\
h_{j} & \equiv \frac{a_{j}}{k^{2}-2 A^{2} a_{0}}(j=1,2,3,4), \\
a_{0} & \equiv \gamma_{0}+2 c_{E} A^{2}\left[\gamma_{0}^{4}+3 \gamma_{0}^{2} \gamma_{1}^{2}+(3 / 8) \gamma_{1}^{4}\right], \\
a_{1} & \equiv 2 A^{2} \gamma_{1}\left[1+2 c_{E} A^{2}\left(4 \gamma_{0}^{3}+3 \gamma_{0} \gamma_{1}^{2}\right)\right], \\
a_{2} & \equiv 2 c_{E} A^{4} \gamma_{1}^{2}\left(6 \gamma_{0}^{2}+\gamma_{1}^{2}\right), \\
a_{3} & \equiv 4 c_{E} A^{4} \gamma_{0} \gamma_{1}^{3}, \\
a_{4} & \equiv\left(c_{E} / 2\right) A^{4} \gamma_{1}^{4} .
\end{array}\right\}
$$




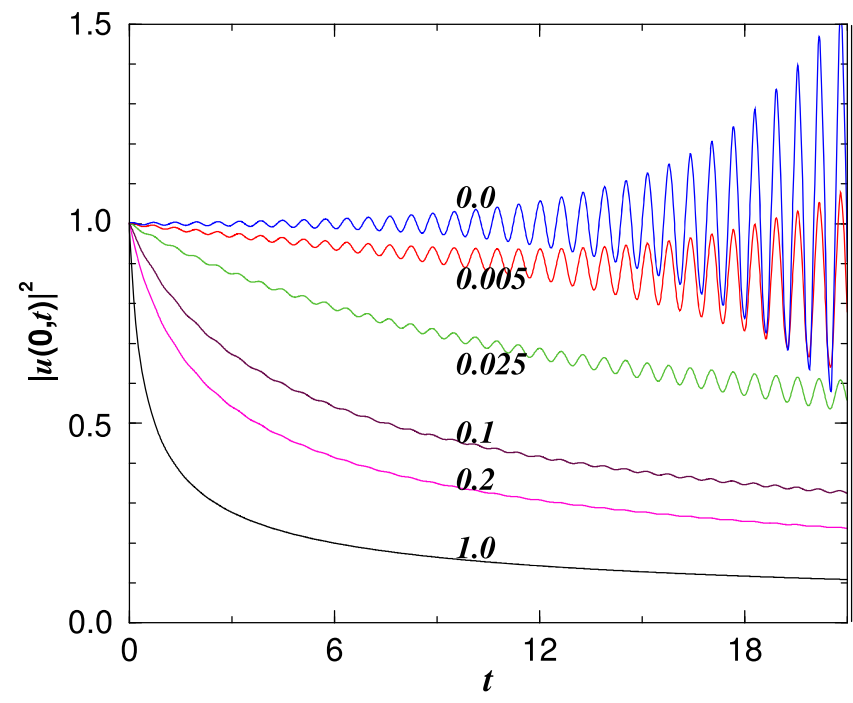

Figure 7. The effect of dissipation in the system. We can exemplify with the results presented in figure 5, by considering $k=3.17$ at the resonant position. For that, in our full numerical calculation, we add in the quintic parameter $g$ and a dissipative imaginary term $\kappa_{3}$, varying it from zero (non-dissipative case shown by the upper results) to $\kappa_{3}=1$ (lower curve), as indicated inside the frame. As expected, the amplitude of the resonance decreases gradually as we increase the dissipation. The other parameters are the same as given in figure 5 , in dimensionless units.

We should also note that in the above equations, for $\kappa_{3} \neq 0$, we have $A \equiv A(t)$, given by equation (11), such that $h_{j} \equiv h_{j}(t)$ and $a_{j} \equiv a_{j}(t)$.

The parametric resonances occur at

$$
\frac{\eta \omega}{2}=\Omega_{1}(\eta=1,2,3,4)
$$

The first parametric resonance, given by $\eta=1$, occurs for $\omega=2\left(\Omega_{1}+\Delta\right)$, where $\Delta$ is detuning. Note that, as expected, all the resonances are absent when $\gamma_{1}=0$, as one can verify in the above expression, where $h_{j}=0$.

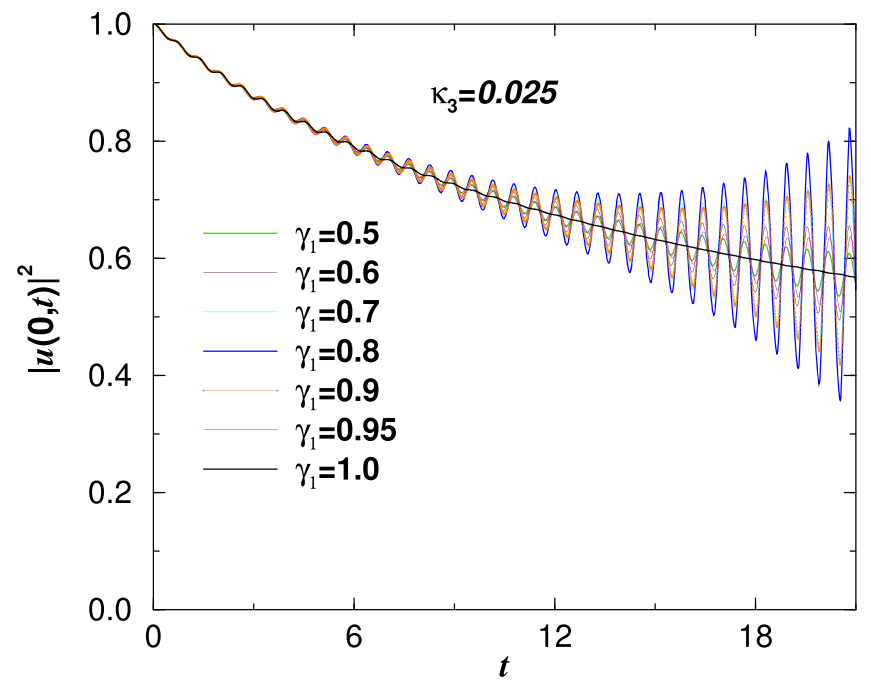

When $a_{0}>0$, which can happen for attractive as well as for repulsive two-body interactions, we obtain

$$
k_{F}=\sqrt{a_{0}} A \sqrt{1+\sqrt{1+\frac{\omega^{2}}{4 A^{4} a_{0}^{2}}}} .
$$

And, in the case that $a_{0}<0$, which can occur for repulsive two-body interactions $\left(\gamma_{0}<0\right)$ when $c_{E}>0$, as well as for attractive two-body interactions $\left(\gamma_{0}>0\right)$ if $c_{E}<0$, the wavenumber is given by

$$
k_{F}=\sqrt{\left|a_{0}\right|} A \sqrt{\sqrt{1+\frac{\omega^{2}}{4 A^{4} a_{0}^{2}}}-1} .
$$

Again we observe that the FW pattern, given by $k_{F}$, will depend on the modulation amplitude of the scattering length, $\gamma_{1}$, in view of the expression for $a_{0}$ given in (31). In the limit of large values for this amplitude and negative $c_{E}<0$ corresponding to the repulsive three-body interactions, we have $k_{F} \sim \gamma_{1}^{2}$, such that the FW period will be given by $L_{F} \sim 1 / \gamma_{1}^{2}$.

For small modulation amplitudes, $h_{1}$, and for three-body losses $\kappa_{3}$, we can perform the analysis based on the perturbation theory. The boundary value for the instability of detuning, $\Delta_{c}$, and the corresponding parametric gain $p_{\max }$, are given by

$$
\Delta_{c}=\frac{\sqrt{h_{1}}}{4 \Omega_{1}} \text { and } p_{\max }=\frac{\sqrt{h_{1}} \omega}{2} .
$$

The threshold value of the amplitude of modulations when the resonance occurs can be found from the condition $h_{1}$ $\left(k=k_{\max }\right)=6 \kappa_{3} A^{4}$. By taking into account that $k_{\max }^{2}=a_{0} A^{2}$, considering $\gamma_{1} \ll 1$, and neglecting the terms $\sim \kappa_{3}^{2}, \gamma_{1}^{2}$ we obtain

$$
\gamma_{1, t h}=\frac{3 \kappa_{3} A^{4} a_{0}}{1+2 c_{E} A^{2} \gamma_{0}^{3}} .
$$

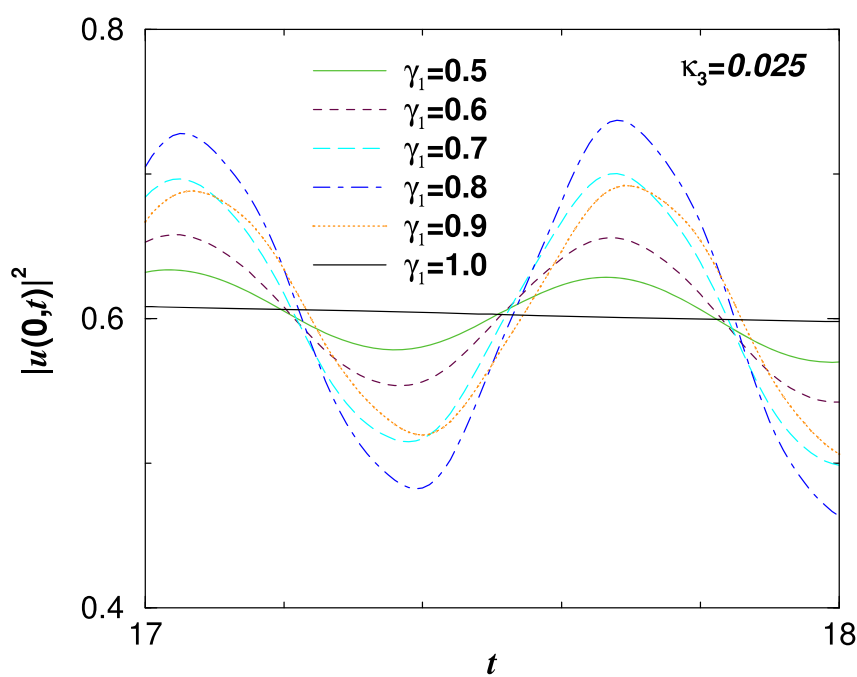

Figure 8. In these two panels we show that the effect of dissipation in the system can be compensated by varying the parameter $\gamma_{1}$. For that, we follow figure 7 , for $k=3.17$, selecting the case where the dissipation parameter is $\kappa_{3}=0.025$. The parameter $\gamma_{1}$ was varied, as shown inside the frame, from 0.5 (same value as in figure 7), to 1 . We notice that the maximum occurs near $\gamma_{1}=0.8$. The panel on the right, for a small time interval, is for easy identification of the different curves. The other parameters are the same as shown in figure 5. 

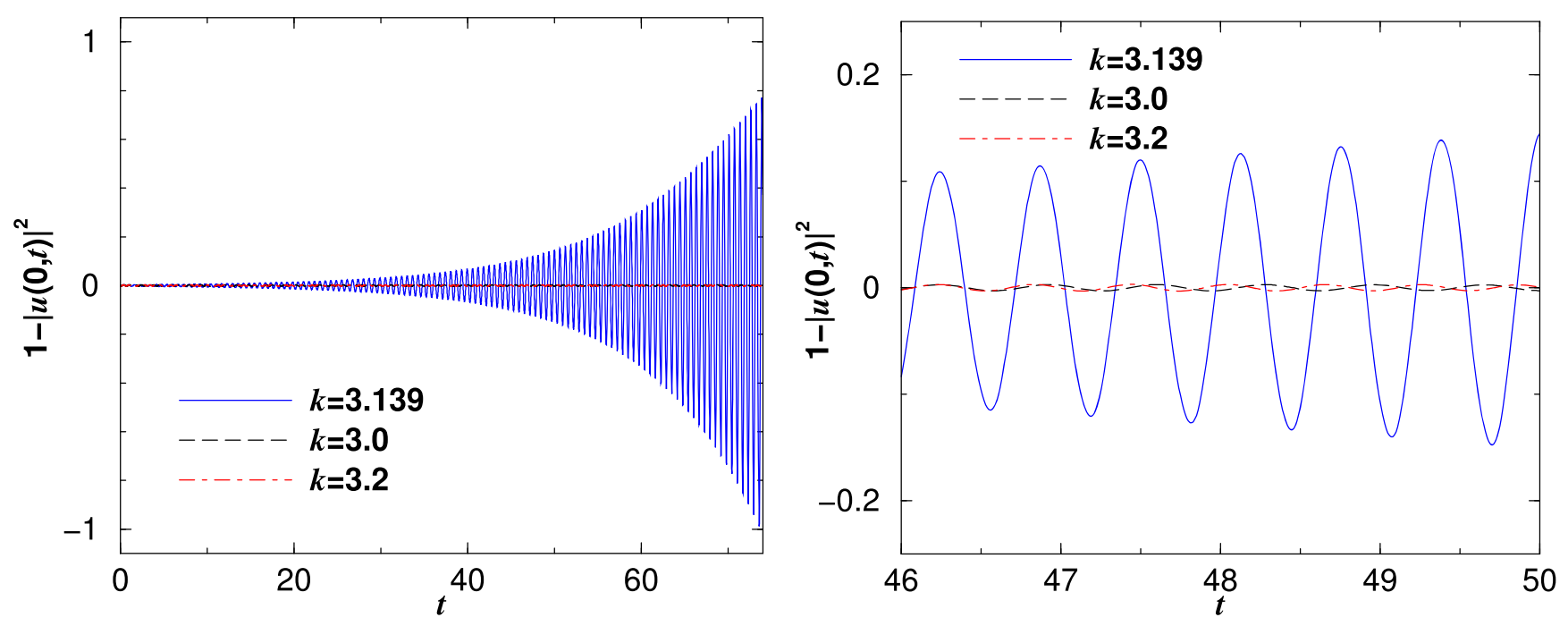

Figure 9. For the repulsive case, with $\gamma_{0}=-0.2$ and $\gamma_{1}=0.2$, from full numerical results, we present the case when the three-body interaction $g(t)$ is given by equation (29) (quartic case). The first parametric resonance for $\omega=20, \epsilon_{0}=0.001, A=1$, and $c_{E}=+1$ (such that $g(t)>0$ ), is found at $k=3.139$ (in agreement with the prediction). In the right panel, for a small interval of time, we show how the amplitude is changing for a small variation of the parameter $k$. All quantities are dimensionless.
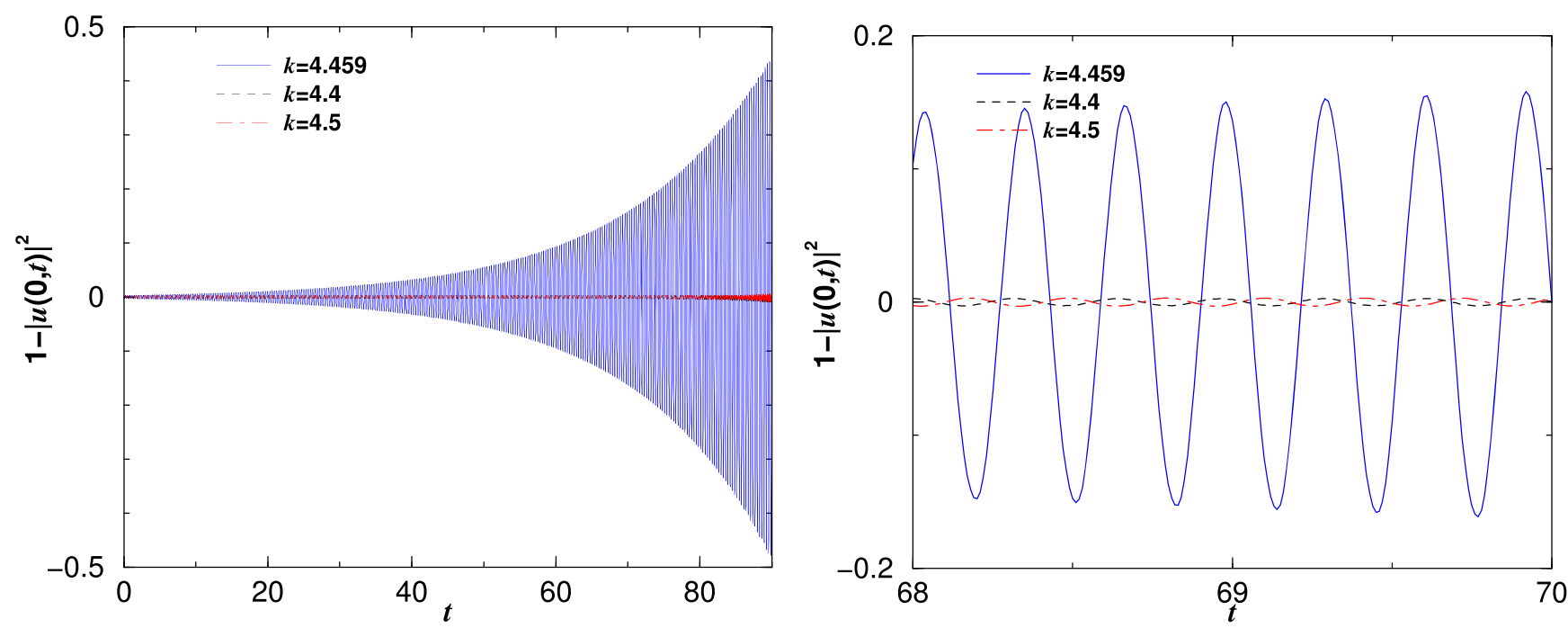

Figure 10. Following figure 9, we show the corresponding second parametric resonance $(\omega=40)$ for the repulsive two-body interaction $\left(\gamma_{0}=-0.2\right)$, when we have a positive three-body parameter $\left(c_{E}=+1\right)$, in the quartic case. The resonance, as predicted, appears at $k=4.45$. This is shown by comparing with results obtained for $k$ smaller and larger than this value, when the oscillation patterns remain almost constant (see right panel). In this case, as compared with figure 9, the peak of the resonant value is manifested for larger values of $t$. All quantities are dimensionless and, except for $k$ and $\omega$, the other parameters are given in figure 9.

In numerical simulations, with $A=\gamma_{0}=c_{E}=1$, leads to $\gamma_{1}$ ${ }_{t h}=1.8 \kappa_{3}$. We can look for $\kappa_{3}=0.01,0.03$.

The next resonance occurs at $\eta=2$, with $\omega=\Omega_{1}+\Delta_{1}$, with the wavenumber

$$
k_{F}=\sqrt{a_{0}} A \sqrt{1+\sqrt{1+\frac{\omega^{2}}{A^{4} a_{0}}}} .
$$

In the particular case of $\gamma_{0}=0$, we have $a_{0}=a_{0}^{0}=3 c_{E} \gamma_{1}^{4} / 8$ and

$$
k_{F}^{0}=k_{F}\left(a_{0}^{0}\right)
$$

In this case, the boundary value of instability of detuning $\Delta_{1 \mathrm{c}}$ and the corresponding parametric gain $p_{\max }$ are given by

$$
\Delta_{1 c}=\frac{\sqrt{h_{2}}}{2 \Omega_{1}} \text { and } p_{\max }=\frac{\sqrt{h_{2}} \omega}{2} .
$$

The perspective on studying FW resonances with three-body parameters having a quartic dependence on the $s$-wave twobody scattering length $a_{s}$, can happen when $a_{s}$ is negative (unbound two-body states) and very large, near the Efimov limit (where the number of three-body bound states, as well as three-body resonances, are expected to increase as the system approaches the unitary limit $\left|a_{s}\right| \rightarrow \infty$ ) [37]. Near this limit, the three-body parameter goes with a fourth power of $a_{s}$ and 

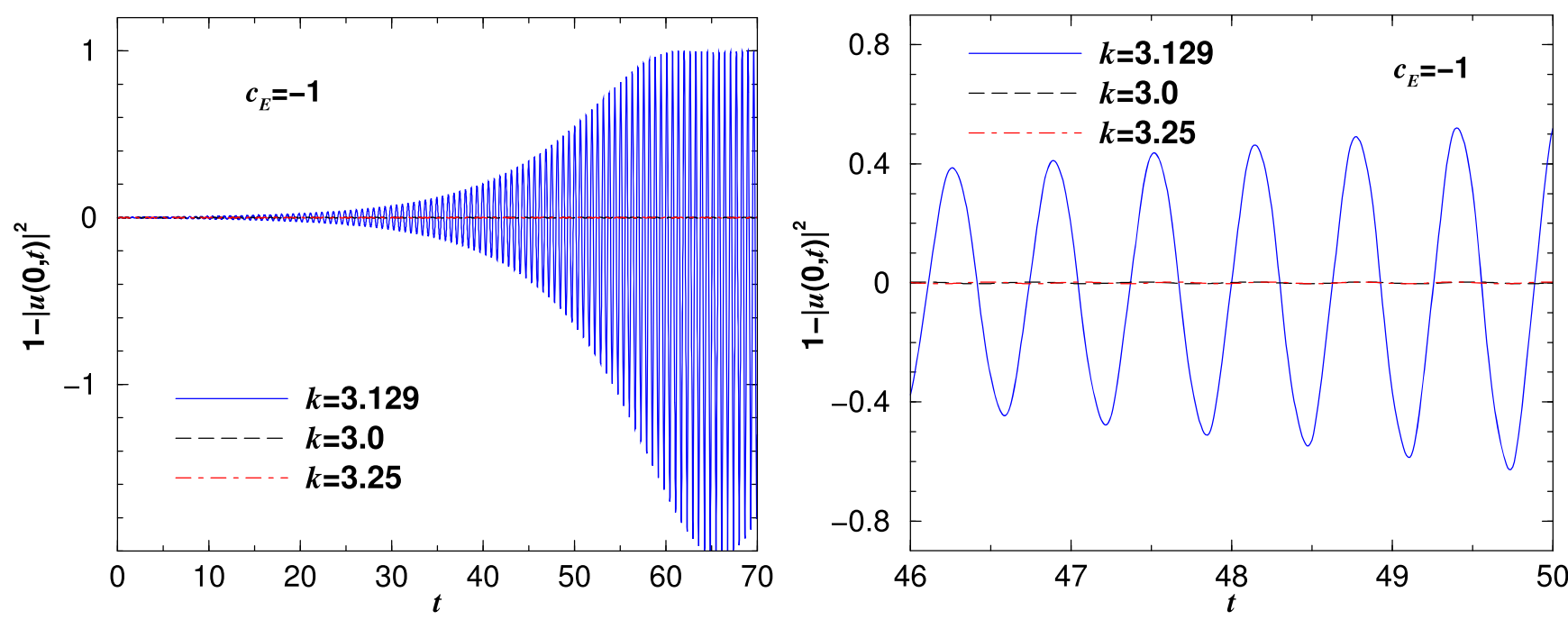

Figure 11. This figure presents the first parametric resonance, with $\omega=20$, for the quartic case (see equation (29)) with repulsive two-body interaction $\left(\gamma_{0}=-0.2\right)$, following the same dimensionless parametrisation as in figure 9 , except that the sign of the three-body parameter $g(t)$ is inverted with $c_{E}=-1$. The resonance, as predicted, appears at $k=3.129$, with the right panel showing that density oscillation remains almost constant for smaller and larger values of $k$. By comparing with figure 9, the resonance is manifested at smaller values of $t$ in the case of $g(t)<0$

can be positive or negative. The corresponding contribution of three-body collisions to the ground-state energy is proportional to the three-body parameter and density $(\sim g$ $n^{3}$ ). In this case, for repulsive $g$, one can find a stable ground state [3]. Figure 3 shows the evolution of the central densities for the second parametric resonance, which occurs at the theoretical predicted value, $k=4.5$.

\section{Numerical simulations}

First, we consider the case when the strength of the threebody interaction is related to the square of the two-body scattering length $a_{s}$, and we present our results in figures 2-4. The emergence of a parametric resonance is displayed in figure 2, for some specific dimensionless parameters, with the modulated two-body parameter given by $\gamma_{0}=0, \gamma_{1}=0.5$ and $\omega=20$. The amplitude $A$ and three-body parameter $c$ are fixed to one, as indicated in the respective captions. The results were obtained for $\left(1-|u|^{2}\right)$ in the central position, as a function of time, by varying the wave number $k$.

The dependence of the spatial period of the Faraday pattern, $L_{F}$, on the frequency of modulations is presented in figure 4 , for the first and second resonance. As shown, the analytical predictions given by equations (18) and (20) are in good agreement with full numerical calculations.

By considering the second model, when the strength of the three-body interactions is proportional to the fourth power of the two-body scattering length, we present the results of numerical simulations in figures 5-11. As we have considered in the first case, given in figures 2 and 3, for this second case the evolution of the central density with the time are plotted in figures 5 and 6 , considering the first and second parametric resonance, respectively. The theoretical predictions for the positions of the resonances are quite well reproduced by the numerical results. The growth rate for the second resonance is shown to be much slower than for the first one.

The influence of dissipation, due to inelastic three-body interactions on the process of the Faraday pattern generation, is presented in figure 7, considering the case for the resonance value $k=3.17$, which was shown (without dissipation) in figure 5. It is observed that the amplitude of the resonance decreases gradually with increasing of the dissipation, as expected. In this figure, the value of the dissipation parameter, $0<\kappa_{3}<1$, is presented inside the frame close to the corresponding plot. In figure 8 , the observed results are demonstrating the existence of a threshold in the amplitude of the modulations, given by $\gamma_{1}$. To verify that, we have selected from figure 7 the case of $\kappa_{3}=0.025$.

In case of repulsive interactions $\left(\gamma_{0}<0\right)$, we first present modelling results for the first and second parametric resonance in figures 9 and 10, by considering that the three-body parameter is attractive, such that $c_{E}>0$ in equation (29). The corresponding predicted values, $k=3.14$ (first resonance) and 4.46 (second resonance), are confirmed by the numerical simulations. The growth rate, for the second resonance, is again lower than the case of the first parametric resonance. The results, in both the cases (first and second resonance) are compared with two values of $k$ outside of the position of the resonance. In the second panels of figures 9 and 10, as in some of our previous results, we show small intervals in time of the respective results shown in the first panels, for better identification of the plots.

As a final result, in figure 11 , we also find it useful to present one model result for the quartic case with repulsive two-body interaction when the three-body interaction is also repulsive, such that $c_{E}<0$ in equation (29). In this case, only the first parametric resonance is shown, with $\omega=20$ and $c_{E}=-1$, considering that the resonant position is very well defined by the analytical expression, $k_{F}=3.129$, with results 
similar to the ones presented in figure 9, such that the second resonance position can be easily predicted by using the corresponding analytical expression. As shown, the resonant pattern grows faster in the case of repulsive three-body interaction.

In the above full numerical results presented in this work, the simulations were performed by using a split-step fastFourier-transform algorithm, with boundary conditions sufficiently extended to avoid reflection effects on the evolutions. In order to facilitate the emergence of Faraday patterns, we started from a uniform density profile of modulus one, adding a small perturbation with the form $u_{\text {initial }}=1+\epsilon_{0}(1+i) \cos (k x)$. The results obtained for the evolutions of the density profiles are quite stable numerically, such that one can easily verify the resonance positions. As a final remark on the present numerical approach, supported by our comparison of results obtained for figures 9 and 11, in the quartic case with repulsive two-body interaction, it is worthwhile to point out that we found the resonant behaviour more stable for longer evolution times when considering repulsive three-body interactions than in the case with attractive three-body interactions.

\section{Conclusion}

In this work we have investigated the generation of Faraday patterns in a BEC system by engineering time-dependent three-body interactions. Two models were analysed, according to the mechanism of modulation and behaviour of the three-body interaction with respect to the atomic scattering length $a_{s}$. First, we considered the strength of the three-body interaction as related to the square of $a_{s}$, supported by the model of [8]. Next, we studied the generation of FWs in the condensate when the strength of the three-body interaction is proportional to the fourth power of the atomic scattering length, which is valid for large values of $a_{s}$ near the Efimov limit $[3,4,37]$.

The results of our analysis and numerical simulations show that the time-dependent three-body interaction excites Faraday patterns with the wavenumbers defined not only by $a_{s}$ and modulation frequency, but also by the amplitude of such oscillation. In the case of rapidly oscillating interactions, we derive the averaged GP equation by considering effective attractive three-body interactions. The MI analysis showed that the attractive three-body interaction effects are weakened by the induced modulations of nonlinear quantum pressure. In our analysis we have considered both cases of repulsive and attractive two-body interactions. We also present simulations for repulsive three-body interactions in the quartic case, when it is proportional to the fourth power of $a_{s}$, considering the case of repulsive two-body interaction, where the behaviour of the resonances can be well identified in agreement with predictions. In all the cases the resonance positions can be easily verified with the help of analytical expressions.

For the experimental observation of Faraday waves the case of the repulsive two-body interactions is important, since in the attractive case the initial noise, which can originate from thermal fluctuations, can initiate MI, competing with the parametric one. Analytical predictions derived in the present work are in good agreement with results of numerical simulations, considering the full time-dependent cubic-quintic extended GP equation.

\section{Acknowledgments}

FA acknowledges the support from Grant No. EDW B14-096-0981 provided by IIUM (Malaysia) and from a senior visitor fellowship from CNPq. AG and LT also thank the Brazilian agencies FAPESP, CNPq and CAPES for partial support.

\section{References}

[1] Petrov D S 2014 Phys. Rev. Lett. 112103201

[2] Braaten E and Hammer H-W 2006 Phys. Rep. 428259

[3] Bulgac A 2002 Phys. Rev. Lett. 89050402

[4] Braaten E, Hammer H-W and Mehen T 2002 Phys. Rev. Lett. 88040401

[5] Kohler T 2003 Phys. Rev. Lett. 89210404

[6] Gammal A, Frederico T, Tomio L and Chomaz P 2000 J. Phys. B 334053

Gammal A, Frederico T, Tomio L and Chomaz P 2000 Phys. Rev. A $61051602(\mathrm{R})$

[7] Abdullaev F Kh, Gammal A, Tomio L and Frederico T 2001 Phys. Rev. A 63043604

[8] Mahmud K W, Tiesinga E and Johnson P R 2014 Phys. Rev. A 90 041602(R)

[9] Inouye S, Andrews M R, Stenger J, Miesner H-J, Stamper-Kurn D M and Ketterle W 1998 Nature 392151

Stenger J, Inouye S, Andrews M R, Miesner H-J, Stamper-Kurn D M and Ketterle W 1999 Phys. Rev. Lett. 822422

[10] Chin C, Grimm R, Julienne P and Tiesinga E 2010 Mod. Phys. Rev. 821225

[11] Abdullaev F Kh and Garnier J 2005 Phys. Rev. E 72 035603(R)

[12] Faraday M 1831 Phil. Trans. R. Soc. London 121299

[13] Staliunas K, Longhi S and de Valcárcel G J 2002 Phys. Rev. Lett. 89210406

[14] Engels P, Atherton C and Hoefer M A 2007 Phys. Rev. Lett. 98 095301

[15] Balaz A and NicolinI A 2012 Phys. Rev. A 85023613

[16] Nicolin A I 2010 Physica A 3894663

[17] Nicolin A I, Carretero-González R and Kevrekidis P G 2007 Phys. Rev. A 76063609

[18] Balaz A, Paun R, Nicolin A I, Balasubramanian S and Ramaswamy R 2014 Phys. Rev. A 89023609

[19] Bhattacherjee A B 2008 Phys. Scripta 78045003

[20] Tang R A, Li H C and Hue J K 2011 J. Phys. B 44115303

[21] Capuzzi P and Vignolo P 2008 Phys. Rev. A 78043613

[22] Nath R and Santos L 2010 Phys. Rev. A 81033626

[23] Lakomy K, Nath R and Santos L 2012 Phys. Rev. A 86023620

[24] Abdullaev F Kh, Ogren M and Sørensen M P 2013 Phys. Rev. A 87023616

[25] Rapti Z, Kevrekidis P G, Smerzi A and Bishop A R 2004 J. Phys. B: At. Mol. Opt. Phys. $37 \mathrm{~S} 257$

[26] Abdullaev F Kh, Darmanyan S A, Bishoff S and Sorensen M P 1997 J. Opt. Soc. Am. B 1427

[27] Abdullaev F Kh, Darmanyan S A and Garnier J 2002 Progress in Optics vol 44, ed E Wolf (Amsterdam: Elsevier) pp 303-365 
[28] Armaroli A and Biancalana F 2012 Opt. Express 2025096

[29] Salasnich L, Parola A and Reatto R 2002 Phys. Rev. A 65 043614

[30] Khaykovich L and Malomed B A 2006 Phys. Rev. A 74023607

[31] Efimov V 1970 Phys. Lett. B 33563

[32] Abdullaev F K and Salerno M 2005 Phys. Rev. A 72033617

[33] Sakaguchi H and Malomed B A 2005 Phys. Rev. E 72 046610
[34] Abdullaev F Kh, Abdumalikov A and Galimzyanov R 2007 Phys. Lett. A 367149

[35] Pelinovsky D E, Kevrekidis P G, Frantzeskakis D J and Zharnitsky V 2004 Phys. Rev. E 70047604

[36] Sabari S, Raja R V J, Porsezian K and Muruganandam P 2010 J. Phys. B: At. Mol. Opt. Phys. 43125302

[37] Braaten E, Hammer H-W and Kusunoki M 2003 Phys. Rev. Lett. 90170402 